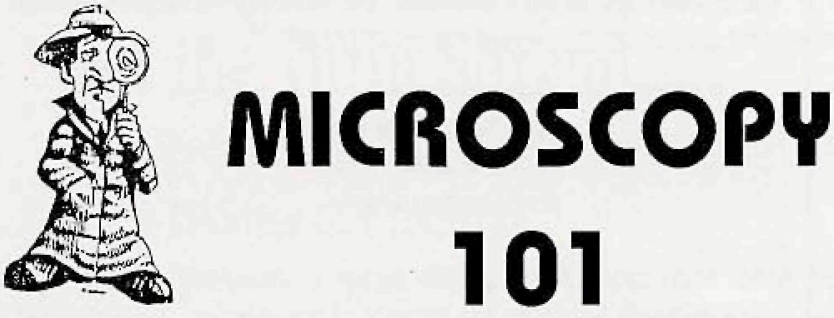

We are pleased to advise that Phit oshel has accepted the responsibitity of Technical Editor for "Microscopy 101". With Phil's management, our plan is to greatly expand this feature. As the feature will continue to cover "approaches" in microscopy, he hopes for YOUR contributions: He with, however, be pleased to receive your "questions" and will seek appropriate answeres. Phit may be contacted at:

oshet@ux1.cso.uinciedu

\section{A New Method of Preparing Specimens for Cryosectioning}

The following technique was developed to circumvent the mess created while preparing tissues for freeze sectioning. In the past, tissues excised from research animals to be freeze-sectioned were placed on a cryostub and covered with O.C.T. mounting medium (Miles Scientific) and placed in a cryostat $\left(-20^{\circ}\right)$ and allowed to freeze. The problem with this technique is that it is messy, and often the tissues slide out of place. Our technique is designed to minimize the mess, while still allowing a quick freeze.

Peel-A-Way disposable plastic tissue embedding molds (Peel-A-Way Scientific) were partially filled with O.C.T. mouriting medium and the excised tissues were placed in them. The tissues are then oriented in the desired way and then covered with O.C.T. This preparation is then placed in a bath of dry ice and acetone $\left(-20^{\circ} \mathrm{C}\right)$ and allowed to freeze solid.

The plastic surrounding the frozen tissue and O.C.T. is then peeled away and the preparation is ready to be mounted onto a cryostub. On a clean cryostub is placed a small amount of O.C.T. mounting medium, and then the tissue block is placed on top of the drop in the desired orientation. The cryostub is then placed into the cryostat and allowed to freeze. Once frozen, the tissue is ready to be sectioned.

One advantage of this procedure is the added protection from drying offered by the plastic Peel-A-Way mold. If the tissues are not immediately used, the capsule can be left on and the entire preparation thing can be wrapped in Parafilm (American National Can) until sectioning is desired.

Travis Phillip Multhaupt, Michigan State University

EMF-1
E-M field cancellation for
scanning electron microscopy
Small, portable device is...
*inexpensive
*effective
*simple to install
*easy to use
*completely safe to use
*a good diagnostic tool

\section{Two Means to Study Cross-Sections of Aluminum Oxide (AL203) Film on an Aluminum Substrate 1) Thin Sectioning}

First trim a small piece of the substrate/film to a needle-like point, then clamping it in a "vise" type specimen holder for an ultamicrotome, and cut (section) in cross section at the fine point.

If the film separates from the substrate, it is possible to also embed a small silver of the specimen in a plastic resin, and to then cut the plastic-embedded specimen. You may need to pre-treat the specimen with a silinization agent to increase the adhesion between the specimen and the resin. This assumes that surrounding the specimen with plastic is acceptable and won't interfere with whatever you need to do in the SEM.

Whether the material is cut naked or embedded in plastic, your friendly local ultramicrotomist should give it a try first with an old diamond knife, cutting at around $30-60 \mathrm{~nm}$ thickness.

Even if the thin sections are not needed, the nice thing about ultramicrotomy is the bulk material becomes well polished during sectioning, presenting an excellent face for EDX and SEM examination.

A diamond knife would do a "great" job of cutting the aluminum and polishing by ultramicrotomy works very nicely. And provided it's done properly, it will NOT destroy the diamond knife.

\section{B. Chiovetti}

\section{2) Mounting for Bulk Polishing}

Glass pieces about $6 \mathrm{~mm}$ thick can be glued onto both sides of the film using 5 minute epoxy. After the glue is hard you can polish as usual for mounted bulk samples, then carbon or gold coat for SEM. Electroplating is also very effective for edge retention, but may not work on a non-conductive film.

Mary Mager, University of British Columbia

\section{SEM Sample Storage}

We have a Hitachi SEM that uses threaded stubs. On the suggestion of Kevin Cronyn (Hitachi sales) I bought plastic hinged-lid boxes (about $4^{\prime \prime} \times 9^{\prime \prime}$ ) from one of the EM suppliers and cut pieces of plexiglass to fit in the bottom. I then drilled and threaded 32 holes in the plexiglass and ran short $\left(-1 / 2^{\prime \prime}\right)$ bolts up through the holes. The thread size is the same as for the Hitachi stubs so you just screw the stubs down on the bolds and set the whole unit in the plastic box. I put one longer $\left(\sim 1^{\prime \prime}\right)$ screw in the middle to act as a handle for getting the plexiglass out of the box. I seem to recall that it came to about $\$ 20$ in supplies for each box as well as two hours of drilling and tapping a bunch of little holes.

For short-term student use I give them Petri plates with double-sided tape in the bottom. Stubs are placed on the tape and stick pretty well. One can get up to 10 or so stubs in one standard Petri dish.

Robert R. Wise, University of Wisconsin Oshkosh

\section{Health Issues With Lyophilized Human Lung Tissue}

A question has recently been raised by Bob Willis of ManTech Environmental concerning the possibility of pathogens surviving in 20 year old lyophilized lung tissue. The samples will require pulverizing for analytical examination, and there is the issue of airborne pathogens being released. As follows is one response:

Pathogens in the specimens may very well still be viable. My major concern would be tuberculosis. Most of the bacteria and viruses that might lurk in old lunds are unlikely to cause serious disease in a person with an intact immune system. TP can cause a serious infection despite a good immune system and infection can be established with very small dose. Pulverization is a perfect way to get aerosols into your lungs. Doing the work in a hood is not a bad idea. You could probably get away with having everyone in the room wear a respirator with a filter fine enough to filter out TB ("N95" respirator). These are available as powered positive air pressure units or as disposable non-powered units. A surgical face mask would not be sufficient. Hepatitis $B$ vaccination shouldn't be necessary, its another bug that probably isn't transmitted by inhalation, but the vaccine is low risk and inexpensive so why not? You might want to look at occupational histories. Some groups, e.g., miners, had higher incidence of TB.

Leon A. Metlay, M.D., University of Rochester Medical Center 